\title{
Creación y funcionamiento del Registro Andaluz de Esquizofrenia
}

RESUMEN: El Registro Andaluz de Esquizofrenia tiene como objetivo principal conocer la prevalencia de la esquizofrenia asistida en el área sur de la provincia de Granada.

PALABRAS CLAVE: registros de casos, esquizofrenia, sistemas de información
SUMMARY: The main aim of the Andalusian Case Register for Schizophrenia is to determine the prevalence of schizophrenia of those cared for by the public network of mental health services in South Granada

KEYWORDS: psychiatry register, schizophrenia, information systems

\section{Introducción}

En el ámbito de la salud mental, la definición más consensuada de lo que es un registro de casos psiquiátricos (RCPs) es aquella que lo considera como un sistema de información longitudinal -centrado en los pacientes- que recoge los contactos de éstos con un conjunto de servicios de salud mental de un área geográfica determinada (1). A partir de esta definición se pueden destacar dos características básicas de los registros acumulativos de casos psiquiátricos en el sentido de que permiten el seguimiento continuo de los pacientes de forma individualizada y que se pueden acumular todos los contactos con los servicios de salud mental, independientemente de la variedad o cantidad de los mismos.

Aunque han existido algunos registros con anterioridad, es a partir de los años 6070 , coincidiendo con el cambio de modelo de atención a la salud mental, cuando surgen numerosos RCPs en diversos países del mundo, como se pone de manifiesto en un inventario publicado en 1986, en el que se enumeran alrededor de 55 registros de casos psiquiátricos (2). Sin embargo, la situación actual ha cambiado respecto al número de RCPs aún en funcionamiento ya que muchos de ellos han dejado de existir debido, entre otros motivos a la falta de financiación y a la entrada en vigor de normativas más estrictas respecto a la confidencialidad de la información. A la vista del cierre de los más antiguos Tansella planteó recientemente si realmente existe la necesidad de establecer y mantener registros de casos psiquiátricos, por lo que se cuestiona si la utilidad que proporcionan justifica sus costes. Sin embargo, para la investigación clínica, epidemiológica y la evaluación de servicios se necesitan sistemas de información que ofrezcan datos con garantía de calidad (3). Por otro lado, el desarrollo de registros acumulativos de casos psiquiátricos se contempla como una necesidad en la mayoría de los planes de salud mental ya que es un instrumento que ofrece información para la evaluación de los procesos de cambio y permite el conocimiento de la población atendida en los servicios de salud mental (4). 
Los principales propósitos para los que han sido creados los RCPs han variado a lo largo del tiempo, desde la investigación clínica y epidemiológica hasta la aplicación de sus resultados en áreas de gestión, evaluación y planificación de servicios $(5,6,7,8)$.

Sin embargo, hay que ser conscientes de las limitaciones que presentan estos sistemas de información, ya que solamente recogen datos de las personas atendidas en los servicios especializados -incidencia/prevalencia administrativa- por lo que no permiten conocer la morbilidad real de la población. Además, la información que ofrecen está muy influenciada por la movilidad geográfica de la población cubierta o por la distribución no uniforme de los servicios de salud mental, que podrían inflar las tasas de prevalencia asistida. Sin olvidar que es un proyecto que supone un elevado coste económico $(9,10)$.

En España, en la década de los 80 surge una corriente de creación de registros acumulativos de casos psiquiátricos como instrumentos para la investigación y evaluación de la asistencia en salud mental (11). Así nació el RCP en la C.A. Vasca en 1984, el cual funciona actualmente de forma independiente en las 3 provincias (4). En la Comunidad Autónoma de Madrid se creó un registro en 1985 que tenía como objetivo cubrir una población de 5.000.000 de habitantes, divididos en 11 áreas sanitarias y 34 distritos (17). Actualmente cubre sólo 5 áreas. El Registro de Casos Psiquiátricos de Asturias funciona desde $1986(1.000 .000$ habitantes aproximadamente) $(13,14,15)$. A partir del año 1987 funciona el RCP de la Comunidad Autónoma de Navarra (16). Aquellos de creación más reciente son el de la C.A. de La Rioja, desde 1990 (263.000 habitantes) y el de la Comunidad Autónoma de Aragón desde 1992 (200.000 habitantes) (17). En Canarias se implantó un registro en el año 1997 y actualmente cubre el $60 \%$ de una población de 1.700 .000 habitantes. Por último, el de la C.A. de Murcia se creó en 1998 y cubre alrededor de 900.000 habitantes. Con una vida más efímera funcionó el de Valencia durante 6 meses del año 1986 (9).

El único registro de casos monográfico sobre esquizofrenia funciona en el área Sur de la provincia de Granada desde el año 1995 (Registro Andaluz de Esquizofrenia), cubriendo una población de 400.000 habitantes aproximadamente (18).

En el resto de las comunidades autónomas españolas existen sistemas de información en salud mental que recogen la actividad de los profesionales del área y funcionan como sistemas de indicadores asistenciales.

Sin embargo, el panorama en nuestro país es todavía desalentador. Aunque existen intentos aislados de poner en marcha dichos sistemas de información, en la mayoría de los casos no reciben al apoyo institucional que necesitan (19) y se encuentran a expensas de la iniciativa de un grupo de profesionales que trabajan de forma "vocacional" para que el registro siga funcionando. Además, todos los sistemas de información existentes han sido creados para responder a finalidades concretas y cuando tratamos de usar la información para algo que no estaba previsto surgen numerosos inconvenientes (20). 
ORIGINALES Y REVISIONES

\section{Justificación de un registro de casos centrado en pacientes esquizofrénicos}

En psiquiatría, existe un creciente interés por vincular la utilización de servicios con los Grupos de Diagnóstico Relacionados (GDR) para posibilitar una adecuada planificación de los recursos sanitarios y la posibilidad de optar entre diferentes estrategias terapéuticas o programas de cuidado. Los datos disponibles se basan, en la mayoría de los casos, en meros recuentos o en fuentes burocráticas o, a lo sumo, se obtienen de estudios no coordinados, los cuales suelen carecer de metodología e instrumentos de evaluación comparables. Por tanto, establecer un registro de casos psiquiátricos, monográfico, es decir, centrado en un grupo de pacientes con un diagnóstico común presenta la ventaja de que la información que se maneja es más homogénea que cuando se refiere a todos los casos psiquiátricos $\mathrm{y}$, por tanto, los objetivos que se pueden alcanzar son más concretos, permitiendo una mayor aplicación de los resultados y mejorando la relación coste-beneficio de este sistema de información.

Del conjunto de problemas de salud cuya atención ha venido siendo competencia de los servicios de salud mental, presenta especial relevancia el que afecta a los pacientes esquizofrénicos. Estos pacientes se han considerado como el grupo diana de la reforma psiquiátrica y el grupo más numeroso para los que se ha transferido la atención desde el hospital a los servicios de salud mental comunitarios. Relevancia que, más allá de su peso relativo en términos de personas afectadas, está en relación con: $i$ )la carga personal y familiar que supone la enfermedad; ii) la carga adicional que supone su tendencia a evolucionar hacia la instauración añadida de un cuadro de cronicidad socialmente invalidante y de marginación social; iii) la posibilidad de prevenir y reducir el desarrollo de este cuadro con medidas integradas sanitarias y sociales.

En enfermedades con una elevada visibilidad como ocurre en la esquizofrenia, en la que alrededor del $70 \%$ de los primeros brotes y casi la mayoría de los esquizofrénicos diagnosticados acuden a un servicio de psiquiatría a lo largo de su vida, la prevalencia administrativa se asemeja a la prevalencia real; por ello, un registro de casos psiquiátricos es un instrumento muy útil para la investigación epidemiológica, ya que existen pocas probabilidades de que los casos se pierdan (5).

Como ya hemos señalado anteriormente, los pacientes esquizofrénicos son enfermos de larga evolución y que suelen permanecer en contacto con los servicios a lo largo de su vida. Por ello, resulta muy interesante conocer su patrón de uso de servicios de forma detallada, así como los episodios de asistencia, que no siempre tienen que coincidir con los episodios de enfermedad. La información sobre el uso de servicios que ofrecen los RCPs se puede representar como un continuo, una «película", y no sólo mediante una «foto» que refleje un corte transversal en el tiempo (21). En el área de la utilización de servicios de salud mental, la mayor aportación de los registros es que ofrecen datos longitudinales, con largos periodos de seguimiento, lo que facilita la observación de las tendencias y además incluyen todos los dispositivos de salud mental de un área determina- 
da. Por tanto, con el cambio de modelo de atención a la salud mental, los estudios llevados a cabo sobre el uso de servicios han evolucionado pasando de aquellos centrados solamente en los ingresos hospitalarios $(22,23,24,25)$ a otros que analizan la utilización de servicios en el marco de la atención comunitaria, por lo que incluyen además los contactos con centros de día y con dispositivos ambulatorios $(18,26,27,28,29)$.

\section{Creación del registro andaluz de esquizofrenia}

A partir de la experiencia de un censo de pacientes esquizofrénicos realizado en el año 1992, y tras un año de preparativos, el registro comenzó su actividad el 1 de Enero de 1995. Su ámbito de cobertura es el área Salud Mental «Granada Sur», con una población de aproximadamente 400.000 habitantes según el Padrón de habitantes del año 1996.

En el año 1995 existían en el área Salud Mental «Granada Sur» una serie de condiciones idóneas para la implantación del registro:

- Territorialización del área de referencia: Mapa Sanitario configurado por 4 distritos sanitarios y 19 zonas básicas de salud que abarcan la totalidad de municipios de la mitad de la provincia.

- Escaso desarrollo de la práctica privada, por lo que es poco probable la pérdida de casos.

- Disponibilidad de datos estadísticos relativos a la población que cubre el área del registro.

- Poca movilidad demográfica por parte de la población que reside en el área de estudio.

- La existencia de un grupo de investigación en salud mental con recursos suficientes para el desarrollo del proyecto.

Los objetivos esenciales del registro son: $i$ ) determinar la prevalencia de la esquizofrenia asistida por la red pública de servicios de salud mental correspondientes al área sur de .la provincia de Granada, a partir del año 1995; ii) describir sociodemográficamente al colectivo de pacientes incluido en el registro; iii) conocer el uso de servicios y el tipo de asistencia que recibe este grupo así como los profesionales implicados en la misma.

Se define como caso aquella persona residente en el área Granada sur, que haya sido diagnosticada de esquizofrenia, trastorno esquizotípico o trastornos de ideas delirantes (CIE-10: códigos F20-F29) y que demande algún tipo de asistencia en la red pública de servicios de salud mental de Granada a partir del 1 de Enero de 1995.

\section{Descripción de la provincia de Granada}

La provincia de Granada se encuentra al sur de España, en la Comunidad Autónoma de Andalucía. Tiene una extensión de 12.531 kilómetros cuadrados y presenta importantes variaciones geográficas, ya que si bien tiene 71 kilómetros de costa en el mar Medi- 
ORIGINALES Y REVISIONES

terráneo, casi la mitad de su superficie está por encima de los 1.000 metros de altitud y cuenta con el pico más alto de la Península Ibérica.

Según el padrón del año 1996, la población de Granada es de 808.053 habitantes; el $49 \%$ son hombres (395.509) y el $51 \%$ son mujeres (412.544). La densidad media de la población es de 63 habitantes por kilómetro cuadrado, siendo más baja que la media nacional. Existe una gran dispersión geográfica de la población que se refleja en el alto número de municipios de la provincia, de los que solamente Granada capital sobrepasa los $\mathbf{2 5 0 . 0 0 0}$ habitantes, siendo el $\mathbf{8 1 \%}$ de ellos menores de 5.000 habitantes. Por tanto, la dispersión de la población en núcleos aislados junto con la existencia de zonas montañosas supone una gran dificultad en la ubicación y distribución de los recursos asistenciales.

Granada cuenta con 4 hospitales públicos ubicados dos en la propia capital (Ciudad Sanitaria Virgen de las Nieves y Hospital Clínico San Cecilio) y otros dos en la provincia (Motril y Baza). Además, en la capital hay tres hospitales privados. El número total de camas en 1991 fue de 3.336, lo que supone una tasa de 4,2 camas por 1000 habitantes, siendo el $88 \%$ públicas y el $12 \%$ privadas. Granada es autosuficiente respecto a los recursos asistenciales especializados para la mayoría de las enfermedades.

Respecto a la atención psiquiátrica, la provincia de Granada está dividida en dos áreas: norte y sur, de $\mathbf{4 0 0 . 0 0 0 ~ h a b i t a n t e s ~ a p r o x i m a d a m e n t e ~ c a d a ~ u n a . ~ A m b a s ~ a ́ r e a s ~ e s t a ́ n ~}$ constituidas por 4 distritos sanitarios en los que se ubica un Equipo Comunitario de Salud Mental que sirve de referencia a toda la población del distrito, y dos Unidades de Salud Mental en sendos hospitales generales, con 30 camas cada una, además de otra Unidad de Salud Mental con 15 camas en un hospital comarcal (Baza). Los dispositivos cuya póblación de cobertura es toda la provincia son la Unidad de Rehabilitación de Área, el Hospital de Día y la Unidad de Salud Mental Infantil y Juvenil. La Unidad de Rehabilitación Intrahospitalaria (Unidad de Media y Larga Estancia), que se encontraba ubicada en el antiguo hospital psiquiátrico cuando comenzó el Registro acaba de cerrarse y se han abierto, en sendas áreas una Unidad de Media y Larga Estancia con 15 camas cada una.

\section{Fuentes de información}

Los dispositivos de salud mental implicados en el Registro son los siguientes: los cuatro Equipos de Salud Mental de Distrito del área Granada Sur (La Alpujarra, Loja, Granada Sur y Costa), la Unidad de Salud Mental del Hospital Clínico San Cecilio, la Unidad de Rehabilitación de Área y la Unidad de Rehabilitación Intra-hospitalaria (Unidad de Media y Larga Estancia).

\section{Datos incluidos}

Una de las características que debe cumplir la información recogida en los RCPs es la de incluir el menor número posible de variables sociodemográficas y clínicas ya que la 
potencialidad de este sistema de información reside en el seguimiento de los pacientes mediante sus contactos con los servicios del área que cubren.

La información que se recoge en el registro se puede dividir en dos grandes grupos. Uno se refiere a los datos epidemiológicos y clínicos, que incluye: sexo, fecha de nacimiento, municipio de residencia, zona básica de salud, tipo de convivencia, nivel educativo, estado civil, situación laboral, diagnóstico principal y fecha del diagnóstico principal. En otro grupo se recoge la información sobre el seguimiento (contactos con los servicios), la cual se obtiene a partir de los contactos que establece el usuario, es decir, de los distintos actos asistenciales que se realizan día a día en los servicios de salud mental.

Para recoger la información referente al uso de servicios de salud mental comunitarios y con centros de día se utiliza la Ficha de Contacto con los Equipos Comunitarios de Salud Mental, la cual es una adaptación de la ficha Mannheim Service Recording Sheet (30). En ella figuran los tipos de intervenciones que se describen en la Clasificación Internacional de Cuidados en Salud Mental $(31,32)$ (anexo 1). En dicha ficha se incluye, de cada paciente, la fecha en la que se realiza el contacto con el dispositivo, el tipo de intervención así como el profesional y el lugar en el que se facilita.

Las variables que se recogen a partir de los ingresos hospitalarios son la fecha del ingreso; la fecha del alta y el diagnóstico clínico al alta.

procedimiento de recogida y procesamiento de la información

La información es recogida principalmente por los profesionales de salud mental de los diferentes dispositivos y enviada posteriormente al registro. En aquellos casos en los que falta información es el personal del registro el que acude a los servicios a consultar las historias clínicas y completar los datos.

A partir de la información sobre la identificación del caso se comprueba si el paciente está ya registrado o es un caso nuevo. En el primer supuesto, se confirma que los datos básicos sean concordantes con los ya existentes y se completa la información faltante. Si el paciente es un caso nuevo, se le asigna un número secuencial y se introduce en la base de datos del registro. A cada paciente se le acumulan los sucesivos contactos con los servicios del área.

Por tanto, la secuencia que sigue la información hasta que se analiza es la que figura en el diagrama 1. Los servicios de salud mental envían los datos al registro y allí es donde se recoge, clasifica, almacena, supervisa y analiza para la posterior devolución de los resultados elaborados a los profesionales que han enviado la información al Registro.

Para preservar la confidencialidad de la información se ha elaborado un «código de conexión", que evita la identificación nominal de los pacientes. En aquellas situaciones en las que es necesario utilizar los nombres para identificar los casos, la información sensible 
ORIGINALES Y REVISIONES

se archiva en una base de datos diferente, manteniendo en ambas bases el número de registro como campo común para relacionarlas.

El control de calidad de la información se ha llevado a cabo mediante una minuciosa depuración de los datos: se realizan controles para detectar posibles duplicados y errores de inconsistencia de la información; se contrastan periodicamente los datos en soporte papel con los informatizados. Tras este laborioso proceso se puede garantizar la exactitud de la información que se ofrece.

\section{Análisis de la información}

Uno de los temas menos desarrollados en la mayoría de los registros de casos es la explotación de los datos. En ocasiones, la cotidianidad de la búsqueda de información deja poco lugar a la producción de resultados elaborados y su posterior utilización, constituyéndose los registros en ocasiones en grandes bancos de datos con una amplia potencialidad, pero escaso rendimiento.

Los resultados que se ofrecen a partir del Registro Andaluz de Esquizofrenia se pueden dividir en datos sobre morbilidad (pacientes) y sobre utilización de servicios (contactos).

a) Morbilidad: Los datos sobre la prevalencia asistida de la esquizofrenia y trastornos afines se presentan mediante tasas brutas y estandarizadas por edad y sexo. Estas últimas han permitido comparar los resultados obtenidos entre las diferentes áreas geográficas que cubre el registro. La información epidemiológica y clínica permite elaborar el perfil sociodemográfico y clínico de los pacientes estudiados.

b) Utilización de servicios: El uso de servicios incluye los servicios ambulatorios, centros de día y unidades hospitalarias; de todos ellos se conoce la fecha en la que se realiza cada contacto. Por tanto, además del número de contactos y del tipo de los mismos (ambulatorios, con centros de día e ingresos psiquiátricos), se puede conocer la distribución de los mismos a lo largo del periodo de seguimiento.

Anualmente se realiza un análisis descriptivo de la información básica del registro, en el que se incluyen datos sobre morbilidad y uso de servicios de toda el área, desagregado por áreas geográficas y por dispositivos asistenciales. $\mathrm{El}$ informe se presenta y se discute con los profesionales de los servicios de salud que han facilitado la información al Registro para una mejor interpretación de los resultados. Además, se elaboran informes específicos para aquellos profesionales del área que lo solicitan.

\section{Investigación en el ámbito del registro}

Como se ha podido comprobar, el registro se perfila como un instrumento metodológico que facilita la investigación epidemiológica, por lo que se han desarrollado diferentes líneas de trabajo dentro de su marco de referencia. Los trabajos se pueden divi- 
dir en dos grupos: uno de ellos incluye aquellos trabajos relacionados directamente con la metodología y los resultados del propio registro y en otro grupo se encuentran aquellos proyectos que han seleccionado sus muestras a partir de los casos incluidos en el registro.

Dentro del primer nivel se incluye una línea de investigación que tiene como objetivo el análisis de la información del uso de servicios bajo tres enfoques diferentes (transversal, longitudinal y dinámico) y que permite la «caracterización del uso de servicios de salud mental por parte de pacientes esquizofrénicos mediante índices de consumo y modelos dinámicos» (FIS: 98/0871) (21,33). Se ha aplicado un análisis de supervivencia al tiempo transcurrido entre los contactos ambulatorios (18), y análisis de Markov para calcular las probabilidades de transición entre los diferentes tipos de contactos (33).

Otro de los proyectos en marcha tiene como objetivo conocer "la prevalencia puntual de personas con enfermedad esquizofrénica en la comúnidad" (FIS 00/724). Este estudio va a permitir comparar la prevalencia real de la esquizofrenia con la prevalencia "asistida" para un distrito sanitario incluido en el registro, lo que ofrecerá información sobre el número de casos "perdidos" por el registro y, por tanto, por el sistema sanitario.

Dentro del segundo grupo se incluyen los estudios de investigación en los que se han seleccionado muestras de pacientes a partir de los casos del registro:

- «Estudio geográfico de una población de esquizofrénicos y de sus necesidades en un área de Granada» (Tesis Doctoral, Rosales Varo, 1999).

- "Análisis del coste de la esquizofrenia según un modelo de prevalencia asistida» (PSICOST-2) FIS-97/1298.

- «Evaluación del coste de la esquizofrenia asociado al uso de servicios» (Tesis Doctoral, Saldivia Bórquez, 2000).

- «Evaluación de la carga familiar en la esquizofrenia". FIS-98/0075-02.(Tesis Doctoral, Bustillo Gálvez, en fase de elaboración).

\section{Limitaciones del registro y planes futuros}

Actualmente se ha cubierto una parte muy importante del proceso de establecimiento y consolidación del Registro Andaluz de Esquizofrenia como es el diseño de las fichas y los protocolos para cumplimentarlas, así como el establecimiento de una rutina de trabajo entre el registro y las diferentes fuentes de información.

Sin embargo, aún nos encontramos en los umbrales del desarrollo del proyecto en lo que concierne al proceso de "retroalimentación" de la información, es decir, las devoluciones actualizadas y periódicas a los servicios que facilitan sus datos al registro. Éstas se hacen con cierto retraso y en muchas ocasiones es una información general que no se utiliza para la práctica clínica diaria. Por ello, entre los objetivos más inmediatos está desarrollar un procedimiento que permita, de forma ágil, elaborar informes de rutina que sean de mayor utilidad clínica. 
ORIGINALES Y REVISIONES

Otra de las limitaciones de las que adolece este sistema de información es que no se registran las consultas de urgencias psiquiátricas en el hospital general de aquellos pacientes que cumplen los criterios de caso registrable.

Como se ha apuntado anteriormente, el registro solamente cubre la mitad sur de la provincia de Granada lo que obliga, por un lado, a excluir a todos los pacientes que no residen en dicha área y por otro, ofrece una información parcial a aquellos dispositivos que cubren toda la provincia, ya que solamente se dispone de los datos correspondientes a los pacientes residentes en el área Granada Sur. Dentro de los planes futuros se incluye la ampliación del registro a toda la provincia de Granada lo que permitiría tener una visión más completa de la atención que reciben los pacientes esquizofrénicos en toda la red asistencial de salud mental de la provincia.

\section{Conclusiones}

Tras varios años de funcionamiento el registro está en condiciones de ofrecer datos fiables y consistentes sobre la prevalencia de la esquizofrenia tratada en el área de estudio y de la ruta que siguen los pacientes en su búsqueda de atención. El Registro Andaluz de Esquizofrenia se puede contemplar como un proyecto vivo, en vías de consolidación y expansión y que ofrece información muy valiosa para la investigación epidemiológica de este colectivo de pacientes.

\section{Agradecimientos}

A todos los profesionales de los servicios de salud mental del Área Granada Sur que han facilitado información al registro. A José Luis Martos por su colaboración en el desarrollo informático del registro. A los siguientes organismos e instituciones que han apoyado económica y/o institucionalmente este proyecto:

- Fundación Andaluza para la Integración Social del Enfermo Mental

- Universidad de Granada

- Hospital Universitario "San Cecilio"

- Escuela Andaluza de Salud Pública

- Fondo de Investigaciones Sanitarias

- Instituto Andaluz de Servicios Sociales 


\section{ANEXO 1 \\ REGISTRO DE CASOS CON DIAGNÓSTICO F20-F29 GRANADA SUR}

FICHA DE CONTACTO CON LOS EQUIPOS COMUNITARIOS DE SALUD MENTAL Equipo de Salud Mental de Distrito: Identificación del Caso

$\mathbf{N}^{\circ}$ historia clínica:

\begin{tabular}{|c|c|c|c|c|c|c|c|c|c|c|}
\hline \multirow[t]{2}{*}{ PROFESIONAL } & FECEA & \multicolumn{6}{|c|}{ TIPO DE INIERVENCIÓN } & \multicolumn{3}{|c|}{ be IIITORVGivcló } \\
\hline & 11 & & $\begin{array}{l}02 \\
08\end{array}$ & $\begin{array}{l}03 \\
09\end{array}$ & $\begin{array}{l}04 \\
10\end{array}$ & $\begin{array}{l}05 \\
11\end{array}$ & $\begin{array}{l}06 \\
12\end{array}$ & $\begin{array}{l}1 \\
4\end{array}$ & 5 & 3 \\
\hline & 11 & $\begin{array}{l}01 \\
07\end{array}$ & $\begin{array}{l}02 \\
08\end{array}$ & $\begin{array}{l}03 \\
09\end{array}$ & $\begin{array}{l}04 \\
10\end{array}$ & $\begin{array}{l}05 \\
11\end{array}$ & $\begin{array}{l}06 \\
12\end{array}$ & $\begin{array}{l}1 \\
4\end{array}$ & 5 & 3 \\
\hline & 11 & $\begin{array}{l}01 \\
07\end{array}$ & $\begin{array}{l}02 \\
08\end{array}$ & $\begin{array}{l}03 \\
09\end{array}$ & $\begin{array}{l}04 \\
10\end{array}$ & $\begin{array}{l}05 \\
11\end{array}$ & $\begin{array}{l}06 \\
12\end{array}$ & $\begin{array}{l}1 \\
4\end{array}$ & 5 & 3 \\
\hline & 11 & $\begin{array}{l}01 \\
07\end{array}$ & $\begin{array}{l}02 \\
08\end{array}$ & $\begin{array}{l}03 \\
09\end{array}$ & $\begin{array}{l}04 \\
10\end{array}$ & $\begin{array}{l}05 \\
11\end{array}$ & $\begin{array}{l}06 \\
12\end{array}$ & $\begin{array}{l}1 \\
4\end{array}$ & 5 & 3 \\
\hline & 11 & & $\begin{array}{l}02 \\
08\end{array}$ & $\begin{array}{l}03 \\
09\end{array}$ & $\begin{array}{l}04 \\
10\end{array}$ & $\begin{array}{l}05 \\
11\end{array}$ & $\begin{array}{l}06 \\
12\end{array}$ & $\begin{array}{l}1 \\
4\end{array}$ & 5 & 3 \\
\hline & 11 & & $\begin{array}{l}02 \\
08\end{array}$ & $\begin{array}{l}03 \\
09\end{array}$ & $\begin{array}{l}04 \\
10\end{array}$ & $\begin{array}{l}05 \\
11\end{array}$ & $\begin{array}{l}06 \\
12\end{array}$ & $\begin{array}{l}1 \\
4\end{array}$ & $\begin{array}{l}2 \\
5\end{array}$ & 3 \\
\hline & I & $\begin{array}{l}01 \\
07\end{array}$ & $\begin{array}{l}02 \\
08\end{array}$ & $\begin{array}{l}03 \\
09\end{array}$ & $\begin{array}{l}04 \\
10\end{array}$ & $\begin{array}{l}05 \\
11\end{array}$ & $\begin{array}{l}06 \\
12\end{array}$ & $\begin{array}{l}1 \\
4\end{array}$ & 5 & 3 \\
\hline & I & & $\begin{array}{l}02 \\
08\end{array}$ & $\begin{array}{l}03 \\
09\end{array}$ & $\begin{array}{l}04 \\
10\end{array}$ & $\begin{array}{l}05 \\
11\end{array}$ & $\begin{array}{l}06 \\
12\end{array}$ & $\begin{array}{l}1 \\
4\end{array}$ & $\begin{array}{l}2 \\
5\end{array}$ & 3 \\
\hline & 1 & & $\begin{array}{l}02 \\
08\end{array}$ & $\begin{array}{l}03 \\
09\end{array}$ & $\begin{array}{l}04 \\
10\end{array}$ & $\begin{array}{l}05 \\
11\end{array}$ & $\begin{array}{l}06 \\
12\end{array}$ & $\begin{array}{l}1 \\
4\end{array}$ & $\begin{array}{l}2 \\
5\end{array}$ & 3 \\
\hline & I & $\begin{array}{l}01 \\
07\end{array}$ & $\begin{array}{l}02 \\
08\end{array}$ & $\begin{array}{l}03 \\
09\end{array}$ & $\begin{array}{l}04 \\
10\end{array}$ & $\begin{array}{l}05 \\
11\end{array}$ & $\begin{array}{l}06 \\
12\end{array}$ & $\begin{array}{l}1 \\
4\end{array}$ & $\begin{array}{l}2 \\
5\end{array}$ & 3 \\
\hline & I & $\begin{array}{l}01 \\
07\end{array}$ & $\begin{array}{l}02 \\
08\end{array}$ & $\begin{array}{l}03 \\
09\end{array}$ & $\begin{array}{l}04 \\
10\end{array}$ & $\begin{array}{l}05 \\
11\end{array}$ & $\begin{array}{l}06 \\
12\end{array}$ & $\begin{array}{l}1 \\
4\end{array}$ & $\begin{array}{l}2 \\
5\end{array}$ & 3 \\
\hline & 1 & $\begin{array}{l}01 \\
07\end{array}$ & $\begin{array}{l}02 \\
08\end{array}$ & $\begin{array}{l}03 \\
09\end{array}$ & $\begin{array}{l}04 \\
10\end{array}$ & $\begin{array}{l}05 \\
11\end{array}$ & $\begin{array}{l}06 \\
12\end{array}$ & $\begin{array}{l}1 \\
4\end{array}$ & $\begin{array}{l}2 \\
5\end{array}$ & 3 \\
\hline
\end{tabular}




\section{ORIGINALES Y REVISIONES}

Tipo de intervención:

$01 \quad$ El problema y su evaluación funcional

02 Establecimiento de relaciones terapéuticas y su mantenimiento

03 Intervenciones psicofarmacológicas y otras intervenciones somáticas

04 Psicoterapia (No sólo dinámica)

05 Cuidados generales de salud

06 Intervenciones dirigidas a la familia, parientes y otros

07 Coordinación de los cuidados

08 (Re)educación de las capacidades básicas, interpersonales y sociales

09 Actividades diarias

10 Asesoramiento y apoyo en gestiones de la vida social

11 Alojamiento alternativo

12 Actividades de capacitación y promoción de empleo

Forma de intervención:

1 Intervención en el dispositivo

2 Visita a domicilio

3 Contacto telefónico

4 Intervención en otro dispositivo

5 No acude 


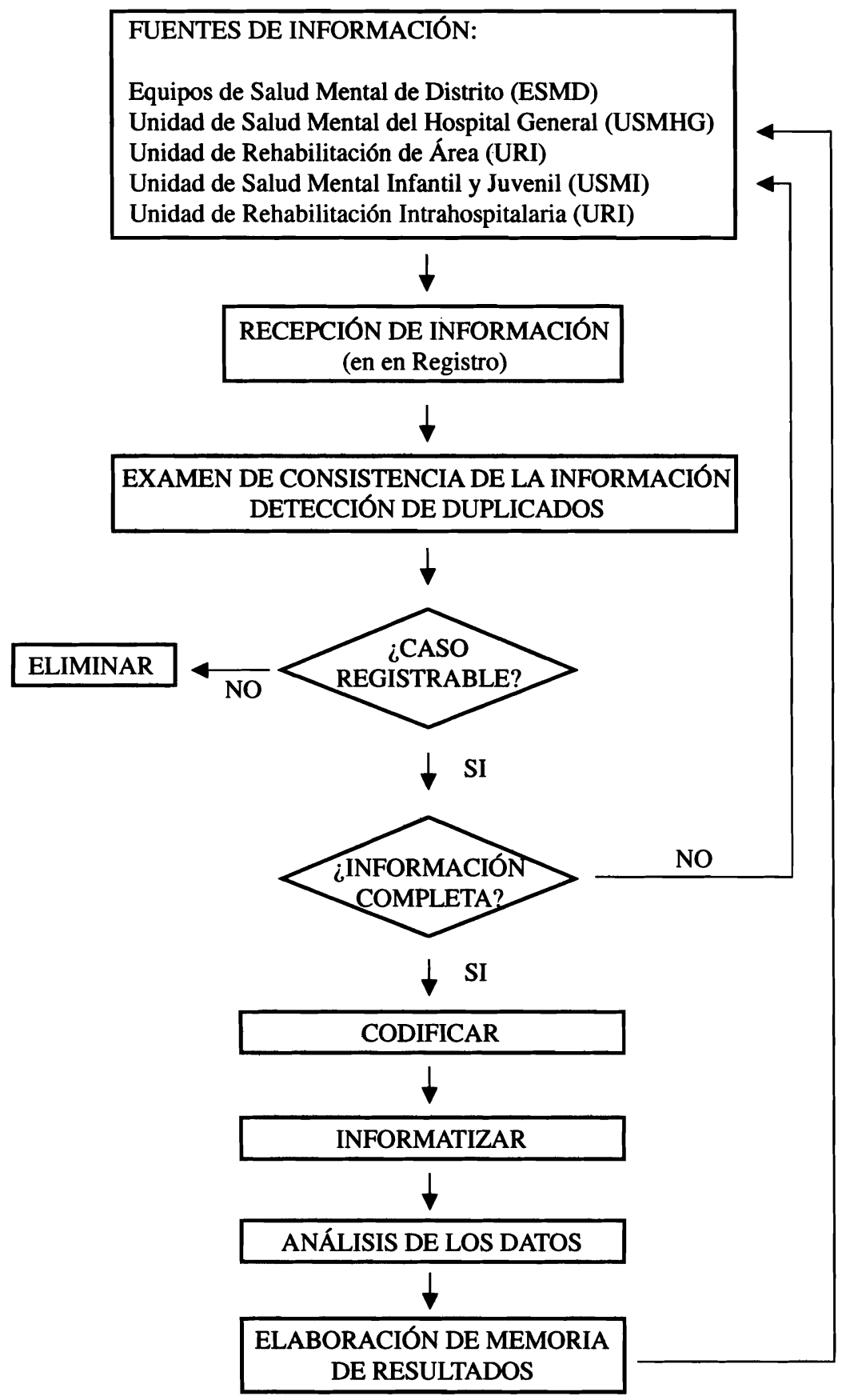


ORIGINALES Y REVISIONES

\section{BIBLIOGRAFÍA}

(1) Horn, G.H.M.M Ten, "Definiciones y clasificaciones: introducción", en Horn, G.H.M.M. Ten, y otros, (eds.) Registro de casos psiquiátricos en salud pública. Un inventario mundial 1960-1985. Santiago de Compostela: Servicio Galego de Saúde 1989, pp.170-173. Publicado originalmente como: Psychiatric case registers in public health. A worldwide inventory, Amsterdam, Elsevier, 1986.

(2) Horn, G.H.M.M Ten, y otros, (eds.), Registro de casos psiquiátricos en salud pública. Un inventario mundial 1960-1985. Santiago de Compostela: Servicio Galego de Saúde 1989. Publicado originalmente como: Psychiatric case registers in public health. A worldwide inventory, Amsterdam, Elsevier, 1986.

(3) Tansella, M., "Do we still need psychiatric case registers?", Acta Psichiatr. Scand., 2000, 101, 4, pp.253-255

(4) Bravo, M.F., y otros, "Hacia una atención comunitaria de salud mental de calidad". Rev. Asoc. Esp. Neuropsiq., Cuadernos Técnicos n²4, Madrid, 2000.

(5) Häfner, H.; Heiden, W. Ander, «The contribution of European case registers to research on schizophrenia», Schizophr. Bull., 1986, 12, pp.26-49.

(6) Wing, J.K. (ed.), Health Services Planning and Research.Contributions from psychiatric case registers, London, Gaskell, 1989.

(7) Eguiagaray, M., "El registro de los casos psiquiátricos", en Aparicio Basauri,V. (coordinador). Evaluación de servicios de salud mental. Madrid, Asociación Española de Neuropsiquiatría, 1993, pp.103-123.

(8) Munk-Jorgensen, P.; Kastrup, M.; Mortensen, P.B., «The Danish psychiatric register as a tool in epidemiology», Acta Psychiatr Scand, 1993, suppl 370, pp.27-32.

(9) Gómez Beneyto, M., y otros, Registro de casos psiquiátricos de Valencia. Valencia, Area de Salut Pública. Diputación Provincial de Valencia, 1989.

(10) Rodríguez-Pulido,F., y otros, «El registro acumulativo de casos en psiquiatría», en González de Rivera y Revuelta, F.; Rodríguez Pulido,F; Sierra López,A. (eds). El método epidemiológico en salud mental, Barcelona, Masson-Salvat Medicina, 1993, pp.151-161.

(11) Pons i Anton, I., y otros, "Los registros acumulativos de caso psiquiátricos como sistema de información y evaluación dentro del marco de la reforma psiquiátrica", Rev. Asoc. Esp. Neuropsiq., 1992, vol XII, 41, pp.115-124.

(12) Fernández Liria, A., y otros, "La implantación del registro de casos psiquiátricos de la Comunidad Autónoma de Madrid", Rev. Asoc. Esp. Neurops., 1994, vol.XIV, 51, pp.569-582.

(13) García González, J., y otros, "El registro de casos de los servicios de salud mental en Asturias: su implantación y utilización para la evaluación asistencial", Rev. San. Hig. Pub., 1988, 62, pp.1469-1482.

(14) Eguiagaray, M., "La creación y utilización del Registro de Casos en Asturias", en García González, J. y Aparicio Basauri, V. (eds.), Nuevos sistemas de atención en salud mental: evaluación e investigación, Oviedo, Consejería de Sanidad y Servicios Sociales, 1990, pp.333-347. 
(15) Pedreira Massa, J.; Eguiagaray, M., «Evaluación de un servicio de psiquiatría infantil a través de un registro acumulativo de casos psiquiátricos», Rev. de psiquiatría Infanto-Juvenil, 1996, 4, pp.63-279.

(16) Mata, I.; Beperet, M.; Madoz,V.; Grupo Psicost, «Prevalencia e incidencia de la esquizofrenia en Navarrar, Anales Sis San Navarra, 2000, vol.23, Supp 1., pp.29-36.

(17) Ferrer Dufol, C., El registro acumulativo de casos de los servicios de salud mental de Aragón, Zaragoza, Departamento de Sanidad y Consumo, 1994.

(18) Moreno Küstner, B.; Luna, J.D.; Torres González, F., «The andalusian case register for schizophrenia: an attempt to obtain patterns of use of mental health services for planning medical care», en Guimón, J.; Sartorius, N. (eds.) Manage or perish: the challenges of managed mental health care in Europe, New York, Kluwer Academicl Plenum Publishers, 1999, pp.573-583.

(19) González Juárez, C., "Sistemas de información en salud mental", Psiquiatría Pública, 1998, 10, 5, pp.271-272.

(20) Gispert, R.; Lesage, A.; Boyer, R. "Los sistemas de información en salud mental. Una puesta al día", Informaciones psiquiátricas, 1991, 123, pp.59-70.

(21) Moreno Küstner, B.; Torres González, F.; Luna del Castillo, J.D., «Analysis of patterns of mental health care with three different approaches (cross-sectional, longitudinal and dynamic)». En prensa

(22) Häfner, H., y otros, «How does gender influence age at first hospitalization for schizophrenia?. A transnational case register study», Psychol. Med., 1990, 19, 4, pp.903-918.

(23) Munk-Jorgensen, P.; Mortensen, P.B.; Machón, R.A., «Hospitalization patterns in schizophrenia. A 13 year follwo up», Schizoph. Res., 1991, 4, pp.1-9.

(24) Eaton, W.W., y otros, "Long-term course of hospitalization for schizophrenia: Part 1. Risk for rehospitalization", Schizoph. Bull., 1992, 18, pp.217-228.

(25) Eaton, W.W., y otros, "Long-term course of hospitalization for schizophrenia: Part 2. Change with passage of time", Schizoph. Bull., 1992, 18, pp.229-241.

(26) Faccincani, C., y otros, "Service utilisation, social support and psychiatric status in a cohort of patients with schizophrenic psychoses. A 7 year follow-up study.", Schizophr Res., 3, 1990, pp.139-146.

(27) Gater, R., y otros, "A comparison of community-based care for schizophrenia in South Verona and South Manchester", Br. J. Psychiatry, 1995, 166, pp.344-352.

(28) Sytema, S.; Micciolo, R.; Tansella, M., "Service utilization of schizophrenic patients in Groningen and South-Verona: An event history analysis", Psychol. Med., 1996, 26, pp.109-119.

(29) Sytema, S.; Micciolo, R.; Tansella, M., "Continuity of care for patients with schizophrenia and related disorders; a comparitive South-Verona and Groningen case register study", Psychol. Med., 1997, 27, pp.1355-1362.

(30) Moreno Küstner, B., y otros, «Ficha Mannheim de contacto con los servicios comunitarios de salud mental», Archivos de Neurobiología, 1997, 60, pp.151-161. 
ORIGINALES Y REVISIONES

(31) Jong, A. de, International Classification of Mental Health Care. Groningen, Department of Social Psychiatry, University of Groningen, 1996.

(32) Torres González, F., y otros, "Clasificación Internacional de Cuidados de Salud Mental", Archivos de Neurobiología, 1997, 60, pp.142-150.

(33) Moreno Küstner, B., Análisis de patrones de utilización de servicios en pacientes esquizofrénicos, Tesis Doctoral, Granada, Universidad de Granada, 1998.

* Psicóloga

** Geógrafa

*** Psiquiatra

Grupo de investigación Salud Mental Granada Sur

Departamento de Psiquiatría, Facultad de Medicina

Universidad de Granada

Correspondencia:

Berta Moreno Küstner

Departamento de Psiquiatría

Facultad de Medicina

Avda. de Madrid, 11

18071 Granada

e-mail:gisamgs@goliat.ugr.es

Fecha de recepción: 9-XI-2000 